\title{
PROPER PSEUDOCOMPACT EXTENSIONS OF COMPACT ABELIAN GROUP TOPOLOGIES ${ }^{1}$
}

\author{
W. W. COMFORT ${ }^{2}$ AND LEWIS C. ROBERTSON
}

\begin{abstract}
A compact Abelian group $G$ admits a strictly finer pseudocompact topological group topology if and only if the weight of $G$ is uncountable.
\end{abstract}

1. Introduction and motivation. Many authors have considered questions concerning increasing or reducing the topology of a topological group without disturbing certain specific features. For example: Can the topology of a locally compact group be enlarged to a locally compact group without augmenting the family of closed subgroups $[12,11,10]$ ? Does every (Hausdorff) group, if not totally bounded, admit a coarser Hausdorff topological group topology $[11,14,6]$ ? Does every totally bounded topological group topology extend to a strictly larger one [2]?

The present paper is a contribution in this vein. Motivated by questions in topological Galois theory $[13,15]$, where pseudocompact groups play a basic rôle [16], and by a fascination with pseudocompact groups as a subject of interest in its own right $[3,20,21,1]$, we show that every compact Abelian group with uncountable weight does admit a strictly finer pseudocompact group topology. Our point of departure is [4], where the question we consider was raised, and answered for totally disconnected groups.

The referee has pointed out that there is a close connection between our result and Theorem 4.4 of Hewitt and Ross [8]. A sharpening of the technique of [8] can be used to prove a result much stronger than that of the present paper, and to demonstrate the existence of finer pseudocompact group topologies of (maximal) weight $2^{2^{\alpha}}$ when $G$ is compact Abelian with $w(G)=\alpha>\omega$. We intend to discuss this in another paper.

In an attempt to make this work reasonably self-contained, we record from [4] those arguments necessary for a complete proof of our theorem.

2. Definitions; Results from the literature. All the topological groups hypothesized in this paper are assumed to satisfy the $T_{0}$ separation property; as is well known (see for example [7, Theorem 8.4]), this guarantees that they are in fact completely regular, Hausdorff spaces, i.e., Tychonoff spaces.

A topological group $G$ is said to be totally bounded if for every nonempty, open subset $U$ of $G$ there is finite $F \subset G$ such that $G=F U$. It is a theorem of Weil

Received by the editors June 26, 1981.

1980 Mathematics Subject Classification. Primary 22C05, 54A10.

Key words and phrases. Compact Abelian group, pseudocompact group.

${ }^{1}$ The authors presented the result of this paper and related results at the Summer Meeting of the Society in August, 1981 in Pittsburgh under the title Compact groups: finer topologies and small dense subgroups.

${ }^{2}$ This author gratefully acknowledges support received from the National Science Foundation under grant NSF-MCS78-00912. 
[19] that every totally bounded group $G$ embeds as a dense subgroup of a compact group $\bar{G}$; further, $\bar{G}$ is unique in the sense that if $\tilde{G}$ is a compact group in which $G$ is dense then there is a function $\varphi$, simultaneously an algebraic isomorphism and a topological homeomorphism, from $\bar{G}$ onto $\tilde{G}$, such that $\varphi(x)=x$ for all $x \in G$. The group $\bar{G}$ is called the Weil completion of the (totally bounded) group $G$.

A topological space $X$ is said to be pseudocompact if every continuous, real-valued function on $X$ has bounded range; for Tychonoff spaces this condition is equivalent to the condition that every locally finite family of nonempty open subsets of $X$ is finite. It is not difficult to show that every pseudocompact group $G$ is totally bounded [3] (and hence is dense in the compact group $\bar{G}$ ). The following result from [3] helps to explain which totally bounded groups are pseudocompact.

2.1. Let $K$ be a compact group and $G$ a dense subgroup of $K$. Then $G$ is pseudocompact if and only if $G$ is $G_{\delta}$-dense in $K$ in the sense that every nonempty $G_{\delta}$ of $K$ has nonempty intersection with $G$.

2.2. LEMMA. Let $G$ and $\tilde{G}$ be compact groups, $\varphi$ a continuous homomorphism of $G$ onto $\tilde{G}$, and $E$ a nonempty $G_{\delta}$ in $G$. Then $\varphi$ is an open function, and $\varphi[E]$ contains a nonempty (compact) $G_{\delta}$ in $\tilde{G}$.

Proof. That $\varphi$ is open follows from the Baire category theorem. The set $E$ contains a set of the form $\bigcap_{n} U_{n}$, with $\operatorname{cl}_{G} U_{n+1} \subset U_{n}$ and $U_{n}$ open in $G$ for $n<\omega$. The set $\bigcap_{n} \varphi\left[U_{n}\right]$, which is $\bigcap_{n} \varphi\left[\operatorname{cl}_{G} U_{n}\right]$, is then as required.

We remark in passing that (as is shown in [4]) it follows without difficulty from (2.2) that if $G$ and $\tilde{G}$ are compact groups, $\varphi$ a continuous homomorphism from $G$ onto $\tilde{G}$ and $\tilde{H}$ a dense, pseudocompact subgroup of $\tilde{G}$, then $\varphi^{-1}(\tilde{H})$ is a dense, pseudocompact subgroup of $G$. In certain contexts this observation makes it easy to define dense, pseudocompact subgroups of a given compact group $G$. Since the result is not needed here, we omit the details of the proof.

In addition to the usual elements of Pontrjagin duality, as set forth, for example, in [7], we need the following algebraic results concerning the p-group decomposition of an Abelian torsion group.

We denote by $P$ the set of prime numbers.

2.3. (See [7, A.3]). Let $G$ be an Abelian torsion group and for $p \in P$ let $G_{p}$ be the set of elements of $G$ whose order is a power of $p$. Then $G_{p}$ is a $p$-primary subgroup of $G$, and $G$ is isomorphic to the group $\bigoplus_{p \in P} G_{p}$.

2.4. (See [7, A.11(III)].) Let $G$ be an Abelian $p$-group with socle $S=\{x \in$ $G$ : order of $x=p\} \cup\{e\}$, for $x \in G$ let $p^{k(x)}$ be the order of $x$ in $G$ and let $L$ be a maximal independent set in $G$. Then $\left\{p^{k(x)-1} x: x \in L\right\}$ is a basis for $S$.

2.5. COROLlary. Let $G$ be an Abelian p-group with $|G|>\omega$ and let $S$ be the socle of $G$. Then $|S|=|G|$.

ProOF. With $L$ as in 2.4 we have $|G| \leq|L| \cdot \omega$ and $|S| \geq|L|$; from $|G|>\omega$ follows $|S|=|G|$, as required.

For a space $X$ and $x \in X$, the symbols $w(X)$ and $\chi(x, X)$, denote respectively the weight of $X$ and the local weight of $X$ at $x$.

The following statements can be substantially generalized, but they are adequate to our needs in their present form.

2.6. (See [7, Theorem 24.15 and 9, 28.58(c)].) Let $G$ be a compact Abelian group with dual group $\hat{G}$. Then $\chi(e, G)=w(G)=|\hat{G}|$. 
3. Proof of the theorem. This first, elementary lemma is a special case of a general topological result noticed by R. M. Stephenson, Jr. [17]; the present proof is taken from [4].

3.1. LEMMA. Let $G=\langle G, \tau\rangle$ be a compact group with $w(G) \leq \omega$ and let $\tau^{\prime}$ be a pseudocompact group topology for $G$ such that $\tau^{\prime} \supset \tau$. Then $\overline{\tau^{\prime}}=\tau$.

Proof. Each point of $G$ is a $\tau^{\prime}-G_{\delta}$ of $G$ and hence of the Weil completion $\bar{G}$ of $\left\langle G, \tau^{\prime}\right\rangle$. It follows that every point of $\bar{G}$ is a $G_{\delta}$. Thus $\chi(p, \bar{G}) \leq \omega$ for all $p \in \bar{G}$ and hence $\bar{G}$ is metrizable (cf. [7, Theorem 8.3]). Thus $\left\langle G, \tau^{\prime}\right\rangle$ is a pseudocompact metric space, hence is compact (see for example [5, Exercise 3D.2]). The identity function from $\left\langle G, \tau^{\prime}\right\rangle$ onto $\langle G, \tau\rangle$ is then a homeomorphism, as required.

For topological groups $G$ and $\tilde{G}$, we write $G \cong \tilde{G}$ if some function from $G$ onto $\tilde{G}$ is both an isomorphism and a topological homeomorphism.

In what follows we denote by $\mathbf{Z}$ and $\mathbf{T}$ the set of integers and the circle, each endowed with the usual algebraic and topological properties. For $p \in P$ the (additive) group of integers $\bmod p$ is denoted $\mathbf{Z}(p)$, and for $0<n<\omega$ we write $\mathbf{T}(n)=\left\{t \in \mathbf{T}: t^{n}=1\right\}$.

DEFINITION. For a cardinal number $\alpha$, a subgroup $K$ of $\mathbf{T}^{\alpha}$ is a $\mathbf{T}$-elementary subgroup of $\mathbf{T}^{\alpha}$ if there is a closed subgroup $S$ of $\mathbf{T}$, with $|S|>1$, such that $K=$ $S^{\alpha}$.

We note that $\mathbf{T}^{\alpha}$ is itself $\mathbf{T}$-elementary in $\mathbf{T}^{\alpha}$. Indeed, the $\mathbf{T}$-elementary subgroups of $\mathbf{T}^{\alpha}$ are exactly $\mathbf{T}^{\alpha}$ and $\mathbf{T}(n)^{\alpha}$ for $0<n<\omega$.

3.2. LEMMA. Let $G$ be a compact, Abelian group with $w(G)>\omega$. Then

(a) there is a subgroup $H$ of $\hat{G}$ such that either $H \cong \bigoplus_{i \in I} \mathbf{Z}_{i}$ with $|I|>\omega$ or $H \cong \bigoplus_{i \in I} \mathbf{Z}(p)_{i}($ for some $p \in P$ ) with $|I|>\omega$; and

(b) there exist $\alpha>\omega$ and a $\mathbf{T}$-elementary subgroup $K$ of $\mathbf{T}^{\alpha}$ and a continuous homomorphism of $G$ onto $K$.

PRoOF. (a) Let $r_{0}$ and $r_{p}(p \in P)$ denote respectively the torsion-free rank and the $p$-rank, and let $M$ denote the injective hull (that is, the minimal divisible extension) of $\hat{G}$. Using (2.6) and [7, Theorems A.14 and A.16] we have

$$
\begin{aligned}
\omega<w(G) & =|\hat{G}| \leq|M|=\omega\left[r_{0}(M)+\sum_{p \in P} r_{p}(M)\right] \\
& =\omega\left[r_{0}(\hat{G})+\sum_{p \in P} r_{p}(\hat{G})\right] ;
\end{aligned}
$$

it follows that either $r_{0}(\hat{G})>\omega$ or there is $p \in P$ such that $r_{p}(\hat{G})>\omega$. In the first case we take

$$
H=\bigoplus_{i \in I} \mathbf{z}_{i} \quad \text { with }|I|=r_{0}(\hat{G}) ;
$$

and in the second case, with $H$ the socle of $\hat{G}_{p}$, we have

$$
\hat{G} \supset H \cong \bigoplus_{i \in I} \mathbf{Z}(p)_{i} \text { with }|I|=r_{p}(\hat{G}) \text {. }
$$

(b) The annihilator $A$ of $H$ in $G$, defined by the rule

$$
A=\{x \in G: \chi \in H \text { implies } \chi(x)=1\},
$$


satisfies $G / A \cong \hat{H}$ (see [7, Theorem 24.11]). When $H \cong \bigoplus_{i \in I} \mathbf{Z}_{i}$ we set

$$
K=\mathbf{T}^{I}=\prod_{i \in I}\left(\mathbf{Z}_{i}\right)^{\wedge}=\hat{H},
$$

and when $H \cong \bigoplus_{i \in I} \mathbf{Z}(p)_{i}$ we set

$$
K=\mathbf{T}(p)^{I}=\prod_{i \in I}\left(\mathrm{Z}(p)_{i}\right)^{\wedge}=\hat{H}
$$

the canonical homomorphism: $G \rightarrow G / A \cong \hat{H}=K$ is then as required.

3.3. LEMMA. Let $G$ be a compact, Abelian group and suppose there is a continuous homomorphism $\varphi$ of $G$ onto a $T$-elementary subgroup $K=S^{\alpha} \subset \mathbf{T}^{\alpha}$ with $\alpha>\omega$. Then there is homomorphism $\Omega$ of $G$ onto $S$ such that the graph of $\Omega$ is a dense, pseudocompact subgroup of $G \times S$.

Proof. We set $\mathbf{T}_{\xi}=\mathbf{T}$ for $\xi<\alpha$, and

$$
W=\bigoplus_{\xi<\alpha} \mathbf{T}_{\xi} \subset \mathbf{T}^{\alpha} .
$$

We define a homomorphism $\psi$ from $W$ to $\mathbf{T}$ by the rule $\psi(t)=\prod_{\xi<\alpha} t_{\xi}$ (this is a finite product for each $t \in W$ ) and, appealing to the divisibility of $\mathbf{T}$, we choose any homomorphism $\tilde{\psi}: \mathbf{T}^{\alpha} \rightarrow \mathbf{T}$ such that $\tilde{\psi} \mid W=\psi$; we define $\bar{\psi}=\tilde{\psi} \mid K$.

It is clear that $\bar{\psi}[K] \supset S$-indeed, $\bar{\psi}[K \cap W] \supset S$. We claim $\bar{\psi}[K]=S$. If $S=\mathbf{T}$ this is clear, and if $S=\mathbf{T}(n)$ then for $t \in K$ we have $t^{n}=1 \in \mathbf{T}^{\alpha}$ and hence $(\bar{\psi}(t))^{n}=1 \in \mathbf{T}$, i.e., $\bar{\psi}(t) \in S$.

Now we define $\Omega=\bar{\psi} \circ \varphi: G \rightarrow S$ and we set $H=$ graph $\Omega \subset G \times S$. It is clear that $H$ is a subgroup of the compact group $G \times S$. To complete the proof it is, by (2.1), enough to show that $H$ is $G_{\delta}$-dense in $G \times S$.

Let $F$ be a nonempty $G_{\delta}$ of $G \times S$. The set $F$ contains a set of the form $E \times\{p\}$ with $E$ a nonempty $G_{\delta}$ of $G$ and with $p \in S$, and from (2.2) (with $\tilde{G}$ replaced by $K)$ it follows that there is a nonempty $G_{\delta} D$ of $K$ such that $D \subset \varphi[E]$. The set $D$ contains a set of the form

$$
\{t(C)\} \times S^{\alpha \backslash C} \subset S^{\alpha}=K
$$

with $C$ a countable subset of $\alpha$ and with $t(C) \in S^{C}$.

Now define $\bar{t} \in K$ by

$$
\begin{array}{rlrl}
\bar{t}_{\xi}=t(C)_{\xi} & & \text { for } \xi \in C \\
& =1 & & \text { for } \xi \in \alpha \backslash C,
\end{array}
$$

set $q=\bar{\psi}(t) \in S$, choose $\eta \in \alpha \backslash C$ and define $\bar{s} \in K$ by

$$
\begin{aligned}
\bar{s}_{\xi} & =\bar{t}_{\xi}=t(C)_{\xi} \in S & & \text { for } \xi \in C \\
& =p q^{-1} \in S & & \text { for } \xi=\eta \\
& =1 \in S & & \text { for } \xi \in \alpha \backslash(C \cup\{\eta\}) .
\end{aligned}
$$

Then

$$
\bar{\psi}(\bar{s})=\bar{\psi}\left(\bar{t} \cdot \bar{t}^{-1} \cdot \bar{s}\right)=q \cdot \psi\left(\bar{t}^{-1} \cdot \bar{s}\right)=q \cdot p q^{-1}=p .
$$

Since $\bar{s} \in\{t(C)\} \times S^{\alpha \backslash C} \subset D \subset \varphi[E]$ there is $\bar{x} \in E$ such that $\varphi(\bar{x})=\bar{s}$, and from

$$
\Omega(\bar{x})=\bar{\psi}(\varphi(\bar{x}))=\bar{\psi}(\bar{s})=p
$$

we have $\langle\bar{x}, \Omega(\bar{x})\rangle \in H \cap(E \times\{p\}) \subset H \cap F$, as required. The proof is complete.

The theorem stated in the abstract is now easily proved. 
3.4 THEOREM. Let $G=\langle G, \tau\rangle$ be a compact Abelian group. There is a pseudocompact group topology $\tau^{\prime}$ for $G$ with $T^{\prime} \supsetneq \tau$ if and only if $w(G)>\omega$.

ProOF. The "only if" statement is Lemma 3.1. For the "if" statement we note from Lemmas 3.2 and 3.3 that there is a homomorphism $\Omega$ of $G$ onto a closed subgroup $S$ of $\mathbf{T}$ with $|S|>1$ such that the set $H=$ graph $\Omega$ is a dense, pseudocompact subgroup of $G \times S$.

Let $\pi$ be the (continuous) projection from $G \times S$ onto $G$ and $\tau^{\prime}$ the topology on $G$ induced by $\pi \mid H$, that is, the topology for $G$ defined by the requirement that $\pi \mid H$ is a homeomorphism of $H$ onto $G$. That $\tau^{\prime} \supset \tau$ follows from the fact that $\pi$ is continuous (from $H$ onto $\langle G, \tau\rangle$ ). The containment is proper because $\langle G, \tau\rangle$ is compact while $H$, a proper, dense subspace of $G \times S$, is not compact.

The proof is complete.

4. Concluding remarks. We address Lemma 3.3 and its proof, retaining the notation used there.

4.1. The homomorphism $\psi: W \rightarrow \mathbf{T}$ given by $\psi(t)=\prod_{\xi<\alpha} t_{\xi}$ is not continuous. Indeed if $\psi$ were continuous then some extension $\tilde{\psi}$ from $\mathbf{T}^{\alpha}$ to $\mathbf{T}$ would be continuous and then $H$, the graph of $\Omega$, would be closed in $G \times S$; this is absurd since $H$ is dense in $G \times S$ and $H \neq G \times S$. Let us note a direct proof that $\psi$ is not continuous. Let $r$ be any element of $S$ such that $r \neq 1$, let $\{\xi(n): n<\omega\}$ be a strictly increasing sequence of predecessors of $\alpha$, and define $t(n) \in W$ by

$$
\begin{aligned}
t(n)_{\xi} & =r \quad \text { for } \xi=\xi(n) \\
& =1 \text { for } \xi<\alpha, \xi \neq \xi(n) .
\end{aligned}
$$

The sequence $t(n)$ approaches the element of $W$ whose coordinates are all 1 , and

$$
\lim _{n} \psi(t(n))=\lim _{n} r=r \neq 1=\psi\left(\lim _{n} t(n)\right) .
$$

4.2. The proof of Lemma 3.3 makes it clear that the graph of $\tilde{\psi}$ is $G_{\delta}$-dense in $\mathbf{T}^{\alpha} \times \mathbf{T}$; this condition is equivalent to the statement that if $C$ is countable subset of $\alpha$ and $t(C) \in \mathbf{T}^{C}$ and $p \in \mathbf{T}$, then there is $\bar{s} \in \mathbf{T}^{\alpha}$ such that $\bar{s}_{\xi}=t(C)_{\xi}$ for $\xi \in C$ and $\tilde{\psi}(\bar{s})=p$. In fact, however, a much stronger statement is true: A value for $\tilde{\psi}$ specified in advance is achieved not only at a point of $\mathbf{T}^{\alpha}$ for which countably many coordinates are specified in advance, but indeed at some point of $\mathbf{T}^{\alpha}$ all but one of whose coordinates are specified. The proof is essentially as before: if $\eta<\alpha$ and $t \in \mathbf{T}^{\alpha \backslash\{\eta\}}$ and $p \in \mathbf{T}$, and if we define $\bar{t}$ and $\bar{s} \in \mathbf{T}^{\alpha}$ by

$$
\begin{aligned}
& \bar{t}_{\xi}=\bar{s}_{\xi}=t_{\xi} \quad \text { for } \xi \neq \eta, \\
& \bar{t}_{\eta}=1, \quad \bar{s}_{\eta}=p \cdot \bar{\psi}(\bar{t})^{-1},
\end{aligned}
$$

then $\tilde{\psi}(\bar{s})=p$. Algebraically, we have for each $\eta<\alpha$ the isomorphism

$$
(\operatorname{graph} \tilde{\psi}) \times \mathbf{T}_{\eta}=\mathbf{T}^{\alpha} \times \mathbf{T} \text {. }
$$

4.3. The fact that $H=\operatorname{graph} \Omega$ is $G_{\delta}$-dense in $G \times S$ shows (since $\{1\}$ is a $G_{\delta}$ of $S$ ) that if $E$ is a nonempty $G_{\delta}$ of $G$ then (kernel $\left.\Omega\right) \cap E \neq \emptyset$. That is, the discontinuous homomorphism $\Omega: G \rightarrow T$ has these properties: Its graph yields a pseudocompact refinement (a covering) of $\langle G, \tau\rangle$, and its kernel is a dense, pseudocompact subgroup of $\langle G, \tau\rangle$. 
4.4. It is known [3] that a topological group is pseudocompact if and only if it is totally bounded and its Weil completion coincides with its Stone-Cech compactification. It follows that for $\langle G, \tau\rangle$ as in Theorem 3.4 the Stone-Cech compactification of $\left\langle G, \tau^{\prime}\right\rangle$ is isomorphic to $\langle G, \tau\rangle \times S$. When $G$ is itself a $\mathrm{T}$ elementary subgroup $S^{\alpha} \subset \mathbf{T}^{\alpha}$ with $\alpha>\omega$, the finer pseudocompact topology $\tau^{\prime}$ we have constructed for $G$ then has the property that the Stone-Cech compactification of $\left\langle G, \tau^{\prime}\right\rangle$ is topologically isomorphic to $S^{\alpha} \times S$ in its usual topology, i.e., to $G$ itself.

\section{REFERENCES}

1. W. W. Comfort and Lewis C. Robertson, Cardinality constraints for pseudocompact and for totally dense subgroups of compact Abelian groups (in preparation).

2. W. W. Comfort and K. A. Ross, Topologies induced by groups of characters, Fund. Math. 55 (1964), 283-291.

3. 483-496.

4. W. W. Comfort and T. Soundararajan, Pseudocompact group topologies and totally dense subgroups, Pacific J. Math. 100 (1982).

5. Leonard Gillman and Meyer Jerison, Rings of continuous functions, Van Nostrand, Princeton, N. J., 1960.

6. Douglass L. Grant, Topological groups which satisfy an open mapping theorem, Pacific J. Math. 68 (1977), 411-423.

7. Edwin Hewitt and Kenneth A. Ross, Abstract harmonic analysis I, Grundlehren der math. Wissenschaften, vol. 115, Springer-Verlag, Berlin and New York, 1963.

8. __ Extensions of Haar measure and of harmonic analysis for locally compact Abelian groups, Math. Ann. 160 (1965), 171-194.

9. __ Abstract harmonic analysis II, Grundlehren der math. Wissenschaften, vol. 152, SpringerVerlag, Berlin and New York, 1970.

10. M. Rajagopalan, Topologies in locally compact groups, Math. Ann. 176 (1968), 169-180.

11. N. W. Rickert, Locally compact topologies for groups, Trans. Amer. Math. Soc. 126 (1967), 225-235.

12. K. A. Ross, Closed subgroups of locally compact Abelian groups, Fund. Math. 56 (1965), 241-244.

13. T. Soundararajan, Galois theory for general extension fields, Crelle J. 241 (1970), 49-63.

14. - Totally dense subgroups of topological groups, General Topology and Its Relations to Modern Analysis and Algebra. III, (Proc. Topological Conf., Kanpur, 1968), Academia, Prague, 1971, pp. 299-300.

15. __ Cohomology of Galois extensions, J. Pure Appl. Algebra 11 (1977), 139-150.

16.

17. R. M. Stephenson, Jr., Pseudocompact spaces, Trans. Amer. Math. Soc. 134 (1968), 437-448.

18. __ Minimal topological groups, Math. Ann. 192 (1971), 193-195.

19. André Weil, Sur les espaces d̀ structure uniforme et sur la topologie générale, Publ. Math. Univ. Strasbourg, Hermann, Paris, 1937.

20. Howard J. Wilcox, Pseudocompact groups, Pacific J. Math. 19 (1966), 365-379.

21. _ Dense subgroups of compact groups, Proc. Amer. Math. Soc. 28 (1971), 578-580.

Department of MATHEMATICS, WEsleyan UNIVERsity, Middletown, ConNECTICUT 06457 